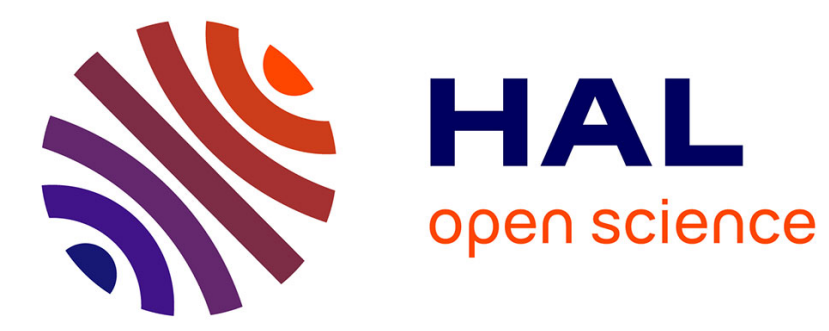

\title{
Phase-shifting digital holographic data compression
}

Meha Hachani, Azza Ouled Zaid, Frédéric Dufaux

\section{To cite this version:}

Meha Hachani, Azza Ouled Zaid, Frédéric Dufaux. Phase-shifting digital holographic data compression. Journal of Optics, 2019, 48 (3), pp.412-428. 10.1007/s12596-019-00538-w . hal-02158838

\section{HAL Id: hal-02158838 \\ https://hal.science/hal-02158838}

Submitted on 10 Jan 2020

HAL is a multi-disciplinary open access archive for the deposit and dissemination of scientific research documents, whether they are published or not. The documents may come from teaching and research institutions in France or abroad, or from public or private research centers.
L'archive ouverte pluridisciplinaire HAL, est destinée au dépôt et à la diffusion de documents scientifiques de niveau recherche, publiés ou non, émanant des établissements d'enseignement et de recherche français ou étrangers, des laboratoires publics ou privés. 


\title{
Phase-shifting digital holographic data compression
}

\author{
Meha Hachani • Azza Ouled Zaid • \\ Frédéric Dufaux
}

the date of receipt and acceptance should be inserted later

\begin{abstract}
Modern holography for 3D imaging allows to reconstruct all the parallaxes that are needed for a truly immersive visualisation. Nevertheless, it possess huge amount of data which induces higher transmission and storage requirements. To gain more popularity and acceptance, digital holography demands development of efficient coding schemes that provide significant data compression at low computation cost. Another issue that needs to be tackled when designing holography coding algorithms is interoperability with commonly used formats. In light of this, the upcoming JPEG Pleno standard aims to develop a standard framework for the representation and exchange of new imaging modalities such as holographic imaging while maintaining backward compatibility with the legacy JPEG decoders. This paper summarize the early work on lossy compression of computer graphic holograms and analyse the efficiency of additional methods that may exhibit good satisfactory coding performance while considering the backward compatibility with legacy JPEG decoders. To validate our findings, the results of our tests are shown and interpreted. Finally, we also outline the emerging trends for future researches.
\end{abstract}

Keywords Holograms $\cdot$ JPEG $\cdot$ region based coding $\cdot$ bit allocation

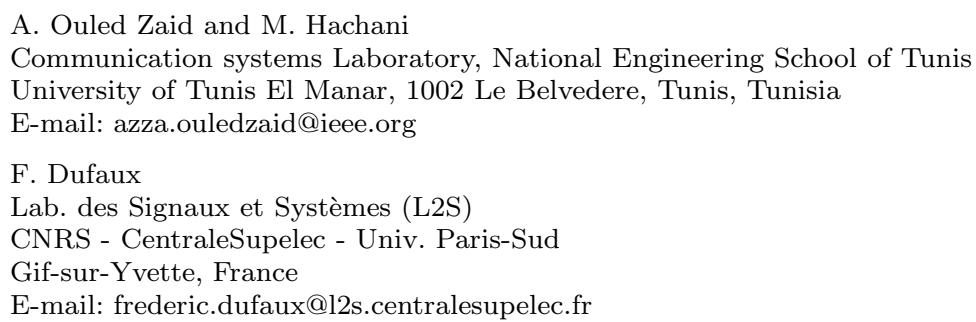




\section{Introduction}

Over the last years, significant advancement has been made in the field of display technology to replace the static planar-like images by immersive volumelike images. Besides the stereoscopic visualization technology [1], there has been an increased interest in holographic technology which allows to reconstruct the $3 \mathrm{D}$ object or scene wavefront directly from the underlying holographic representation. Thank to its intrinsic wave optics, holographic display has the advantage of reproducing object depth through phase and handling anti-aliasing implicitly, thus, allowing to surpass the limitations of image based $3 \mathrm{D}$ visualisation technology.

The optical holography has been initiated in 1947 by Denis Gabor to eliminate aberrations in electron microscopy by recording and reconstructing amplitude and phase of a wave field [4]. Since then, many techniques have been developed to reconstruct and record holograms with the same aim of providing simpler solutions and higher quality [5]. The physical concept of light propagation can be modelled and simulated on a computer, enabling the production of computer generated holograms (CGHs) $[8,9]$ to avoid the need for a physical holographic recording set-up. Considering the convenience of CGH for real objects and virtual objects, Phase Shifting Interferometry (PSI) is an important form of hologram generation techniques that has been proposed to improve reconstruction quality in digital holography.

When taken in its native double-precision format, a digital hologram (DH) requires about hundreds megabytes of storage, whereas higher resolution holograms need gigabytes. Today, the storage of such huge amount of data is not a big issue. But its efficient transmission is still hampered by the lack of network transmission capacities. To overcome this problem, the use of DH compression techniques is mandatory for transmission purpose.

Hologram compression differs to image compression principally because holograms encode 3D information in complex-valued samples and secondly because of their speckle nature. But, due to the fact that digital holographic content represents the diffraction patterns that correspond to $3 \mathrm{D}$ scene information as intensity and/or phase over a bidirectional plane, the most of research work on holographic data compression have focused on the extension of conventional image/video coding algorithms. Fundamentally there are two modes of image compression: lossless and lossy compression. Lossless compression techniques have been already performed on digital holographic data. Even if perfect reconstruction is to be attained, reversible compression methods are usually inefficient for holographic data [10]. This is principally due to the low spatial redundancies of hologram's speckle nature. But beside that, lossy compression of holographic data still play a critical role in reducing the storage and transmission costs [11].

To date, there is no coding standard appropriate for holographic images. Various reviews, summarizing the pioneering work on CGH data compression were published $[13,7,12,14-16]$. On the other hand, the upcoming JPEG Pleno standard is actually working on the issue of high efficient coding of plenoptic 
(a)

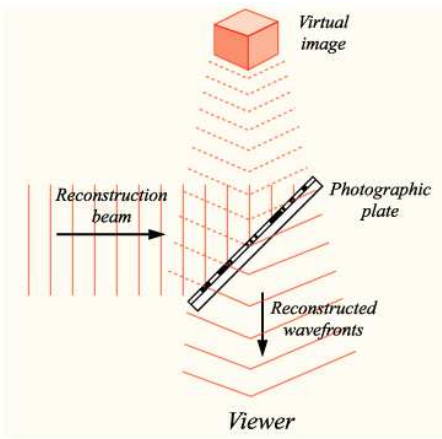

(b)

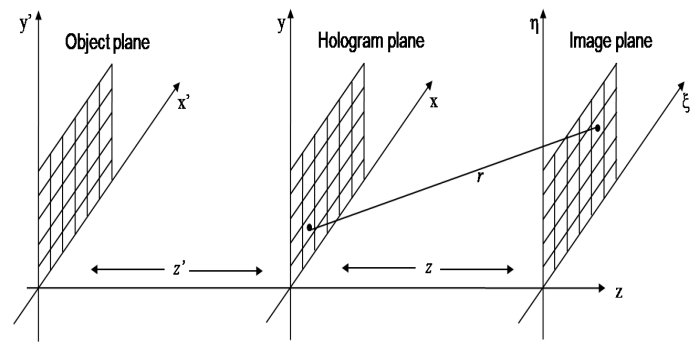

Fig. 1: a) Hologram reconstruction [19]; b) Diffraction geometry for hologram reconstruction.

images and digital holographic data while maintaining backward compatibility with legacy JPEG decoders [17]. It is evident that a common representation framework, which is backward compatible with JPEG would provide interpretability that might enable a wider use of this new type of image modalities without breaking the current JPEG ecosystem.

The aim of this paper is twofold. First, we bring a deep analysis of the most known compression methods, applied to phase-shifting digital holograms (PSDH). Second, we present a JPEG based solution for compressing this type of holographic data. The proposed codec inherently supports region based rate control, offering near-optimal compression performance while maintaining backward compatibility with JPEG. Experimental assessments demonstrate that the proposed coding system yields very competitive compression performance that is sometimes even better than that of wavelet-based techniques and HEVC codecs.

The paper is structured as follows. Section II gives the basic concepts of hologram generation and reproduction. Section III summarizes the pioneering work on lossy compression of PSDH data. Section IV describes the proposed compression algorithm which uses a bit allocation mechanism while guaranteeing a full compliance with the JPEG bitstream. Section IV assesses the efficiency of our proposed method by comparing it to the well known image compression approaches. Finally, Section V presents our conclusions.

\section{Basic concepts in digital holography}

Holography is a lens less imaging process that generate a wave field as a $2 \mathrm{D}$ interference pattern. If a coherent light beam (reference beam) interferes with the light wave diffracted or scattered from an object, then the full information about both amplitude and phase of the diffracted or scattered wave can be 
recorded on a recording medium as a hologram. The object wave can be reproduced by illuminating the hologram with the reference wave and the hologram reconstruction refers to the process of regenerating a $3 \mathrm{D}$ wave field from the hologram.

The evolution of recording devices and computer technologies led to the development of digital holograms that make it possible to use digital setup similar to that of optical hologram generation. When using digital processing for hologram generation, the interference pattern is captured using a CCD (charged coupled device) instead of film. Specifically, a digital hologram is produced when the interference pattern is recorded on the surface of a CCD. The obtained hologram is electronically recorded and stored. Besides, the holography is easier to be processed when the whole generation procedure is carried out numerically with Computer Generated Hologram (CGH). It is worth noting that Digital Holograms are obtained by optical means on a CCD, whereas Computer Generated Holograms are obtained by numerical methods on a computer. Furthermore, the design of Phase-shift Digital Holography (PSDH) gives a good solution for suppressing the unwanted information in the reconstruction procedure.

Throughout this paper we will retain the Phase-shift Digital Holography technique since it guarantees a superior reconstruction quality than those of the other available techniques.

\subsection{Hologram generation and reproduction}

Holograms are recorded under a coherent beam of light and saved on a physical recording medium. A traditional setup involves the recording medium, the object to be recorded, a laser, a beam-splitter (BS), and some lens. The laser is split into two separate beams to create an object wave and a reference one; see Figure 1a for a schematic illustration. The object wave will be used to illuminate the object, and successively illuminate the recording medium. By doing so, some light will be absorbed while the other will be scattered and comes in contact with the recording medium. On the other hand, the reference wave will be used to illuminate the recording medium in which interference is occurred, and the result pattern is recorded onto the medium.

In accordance with Maxwell equations, the complex amplitude of the object wave $U_{O}$ and the reference wave $U_{R}$ at the hologram plane are given by:

$$
\begin{aligned}
& U_{O}=A_{O} \exp \left(j \varphi_{O}\right) \\
& U_{R}=A_{R} \exp \left(j \varphi_{R}\right)
\end{aligned}
$$

where $\left(A_{O}, \varphi_{O}\right)$ and $\left(A_{R}, \varphi_{R}\right)$ are the amplitude and phase distribution pairs of the reference and the object wave respectively. The resulting intensity of 
the interference of the object and reference waves may be expressed as:

$$
\begin{aligned}
I_{H} & =\left|U_{O}+U_{R}\right|^{2} \\
& =\left|U_{O}\right|^{2}+\left|U_{R}\right|^{2}+U_{R} U_{O}^{*}+U_{R}^{*} U_{O}
\end{aligned}
$$

with $*$ the complex conjugate operator. We can see that the interference pattern consists of four terms: the first and second terms correspond to zero-order diffraction, the third one is referred to as conjugate or twin image and the forth one is the virtual image.

The recording medium for holography is commonly some type of film emulsion where the information representing the hologram is stored to be later reconstructed. At a given position $(x, y)$, the recording process is associated to the typically named hologram function $h(x, y)$ (or amplitude transmission of the recording medium), which can be written as:

$$
h(x, y)=h_{0}+\beta \tau I_{H}(x, y)
$$

where $\tau$ is the exposure time, $\beta$ is a constant, and $h_{0}$ is the amplitude transmission of the unexposed medium [6]. In essence, some physical characteristic of the recording medium is modulated by the light intensity, expressed in Eq. 4 , by means $h(x, y)$ transmission function that corresponds to the hologram.

\subsubsection{Hologram reconstruction}

An hologram is a 2D plane consisting of several points (apertures) and the object image is optically reconstructed by illuminating this plane (see Figure 1b). To keep the analysis simple the object surface is supposed to be flat, that means the distance between the original object and hologram plane is approximately constant. The virtual image occurs at the position of the original object and the real image is observed at a distance $z$ from the hologram but in the opposed direction. Notably, the reconstruction procedure is considered as a diffraction phenomenon which is realized when the reference wave illuminates the hologram (an aperture) in an opaque plane. It can be explained by scalar diffraction theory if two fundamental assumptions are met [41]:

1. the diffracting fields must not be observed too close to the aperture, and

2. the diffracting aperture must be large enough compared to the wavelength

By assuming that the reference wave is perpendicular to the hologram plane the obtained object diffraction wave can be calculated using the FresnelKirchhoff integral under the scalar diffraction theory [20]:

$$
U(\xi, \eta)=-\frac{i}{\lambda} \int_{-\infty}^{+\infty} \int_{-\infty}^{+\infty} U(x, y) \frac{\exp (j k r)}{r} d x d y
$$

with

$$
r=\sqrt{z^{2}+(x-\xi)^{2}+(y-\eta)^{2}} .
$$

$U(x, y)$ is the complex amplitude of the real object image, $z$ is the distance between the two planes (real image and hologram plane, respectively virtual 
image and hologram plane), $\lambda$ is the wavelength of the reference wave, $k$ the wave number and $r$ is the distance between point $\mathrm{P}$ with coordinate $(x, y)$ in

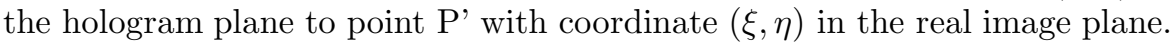

\subsection{Digital holography}

Digital holograms have the similar setup as optical hologram generation. The main difference is that interference pattern is recorded using a charged coupled device (CCD) instead of film. However, the use of CCDs presents certain limits: digital holography does not offer full-parallax and high resolution. Another difficulty arises due to pixelated structures which are not small enough to produce sufficient viewing angles. On the other hand, the sensitivity of CCD cameras is better than that of photographic emulsions used for optical holography. Moreover, digital holograms are able to use numerical reconstruction methods which allow many improvements like suppression of unwanted information and removal of artifacts.

\subsubsection{Numerical reconstruction}

Some approximations have been made to scalar diffraction theory, to reduce the calculation of the diffraction patterns for easier mathematical manipulations. In practice, the Fresnel and Fraunhofer approximations, as well as the Angular Spectrum Method (ASM) are the most commonly used to deal with wave propagation.

Reconstruction by Fresnel and Fraunhofer approximations Within the Fresnel approximation, the wave field is observed from a plane instead of a point passing through an aperture. This facilitates the mathematical manipulations as compared to Rayleigh-Sommerfeld and the Fresnel-Kirchhoff integral by propagating a wave from the object plane to the observer plane without considering each point on the hologram independently [20]. As illustrated in Figure 1b, we are interesting in determining the wave field $U(\xi, \eta)$ at point $\mathrm{P}^{\prime}$ with coordinate $(\xi, \eta)$ in the observer plane due to a field $U(x, y)=I_{H}(x, y) U_{R}(x, y)$ at point $\mathrm{P}$ with coordinate $(x, y)$ in the hologram plane. With regard to the geometrical optics this is the paraxial approximation case, where the ray makes a small angle with respect to the optical axis. According to the diffraction principal, this deals with a near field propagation which is equivalent to a small distance of propagation, $z$.

The Fresnel approximation is obtained by expending $r$ introduced in Eq. 6, as a Taylor series by factoring a $z$ outside the square root in Eq. 7 and retaining only the first two terms of the expansion:

$$
\begin{aligned}
r & =z \sqrt{1+\left(\frac{\xi-x}{z}\right)^{2}+\left(\frac{y-\eta}{z}\right)^{2}} \\
& \approx z\left[1+\frac{1}{2}\left(\frac{x-\xi}{z}\right)^{2}+\frac{1}{2}\left(\frac{y-\eta}{z}\right)^{2}\right]
\end{aligned}
$$


At this stage, we can notice that in the denominator of the integral in Eq. 6 , small error arises from neglecting the second two terms in the expression of $r$. Then, we can approximate the denominator simply as $z$. Inversely, these second two terms in the expression of $r$, appearing in the the exponent are significant, since phase changes of even a fraction can alter the value of this exponential term by a large amount. Thus by keeping the full expression of $r$ in the exponent term and dropping its second two terms in the dominator of the integral, the resulting complex amplitude of the real image is given as:

$$
\begin{aligned}
U(\xi, \eta)= & -\frac{i}{\lambda z} \exp (i k z) \int_{-\infty}^{+\infty} \int_{-\infty}^{+\infty} U(x, y) \\
& \exp \frac{i k}{2 z}\left[(x-\xi)^{2}+(y-\eta)^{2}\right] d x d y
\end{aligned}
$$

By factoring the term $\exp \left(\frac{i k}{2 z}\left(\xi^{2}+\eta^{2}\right)\right)$ outside the integral in 9 , the Fresnel diffraction can be formulated as:

$$
\begin{aligned}
U(\xi, \eta)= & \frac{\exp (i k z)}{i \lambda z} \exp \left(\frac{i k}{2 z}\left(\xi^{2}+\eta^{2}\right)\right) \\
& \int_{-\infty}^{+\infty} \int_{-\infty}^{+\infty} U(x, y) \exp \left(\frac{i k}{2 z}\right)\left[x^{2}+y^{2}\right] \\
= & \exp \left(\frac{-i k}{z}\right)[x \xi+y \eta] d x d y
\end{aligned}
$$

One may easily perceive that the mathematical expression of $U(\xi, \eta)$ is the Fourier transform of the product of the wave field $U(x, y)$ and a complex phase exponential. This formulation is named Fresnel approximation, where the observer is in the near field of the aperture.

By supposing the propagation distance $z$ to be very far, $z>>\frac{k\left(x^{2}+y^{2}\right)}{2}$, we can approximate the quadratic phase factor $\left(\exp \frac{i k}{2 z}\left[(x-\xi)^{2}+(y-\eta)^{2}\right]\right)$ in Eq. 9 as being flat. Then, the quadratic phase factor $\exp \left[\frac{j k}{2 z}\left(\xi^{2}+\eta^{2}\right)\right]$ within the integral in 10 is approximately equal to unity. This brings us to the Fraunhofer approximation (where the observer is located in the far field of the aperture):

$$
\begin{aligned}
U(\xi, \eta)= & \frac{\exp (i k z)}{i \lambda z} \exp \left[\frac{i k}{2 z}\left(\xi^{2}+\eta^{2}\right)\right] \iint_{-\infty}^{+\infty} U(x, y) \\
& \exp \left[\frac{-i k}{z}(x \xi+y \eta)\right] d x d y
\end{aligned}
$$

which can be interpreted as the product of multiplicative phase factors and the Fourier transform of the aperture distribution $U(x, y)$, evaluated at frequencies $f_{\xi}=\frac{\xi}{\lambda z}$ and $f_{\eta}=\frac{\eta}{\lambda z}$.

Due to the performed distance approximations, the Fresnel and Fraunhofer based reconstructions are suitable for longer distances. 
Reconstruction by Angular Spectrum Propagation (ASP) An alternative method to describing the diffraction is given by the ASP also designated by the planewave decomposition. Taking into account the propagation of light from the hologram plane $z=0$ to the image plane at nonzero distance $z$ (see Figure 1b), let's designate by the direction cosines, related to the spatial frequencies $f_{x}$ and $f_{y}$ of the Fourier transform by $\alpha=f_{x} \lambda$ and $\beta=f_{y} \lambda$, respectively. The angular spectrum of the light field $U(x, y ; 0)$ (i.e. that across the hologram $(z=0)$ plane) is given by:

$$
\begin{aligned}
A\left(\frac{\alpha}{\lambda}, \frac{\beta}{\lambda} ; 0\right)= & \iint_{-\infty}^{+\infty} U(x, y ; 0) \\
& \exp \left[-i 2 \pi\left(x \frac{\alpha}{\lambda}+y \frac{\beta}{\lambda}\right)\right] d x d y
\end{aligned}
$$

By analogy, the angular spectrum $\left(\frac{\alpha}{\lambda}, \frac{\beta}{\lambda} ; z\right)$, of the wave field $U(x, y ; z)$ at the image plane $(z>0)$ from the original one $(z=0)$, is given by:

$$
A\left(\frac{\alpha}{\lambda}, \frac{\beta}{\lambda} ; z\right)=\iint_{-\infty}^{+\infty} U(x, y ; z) \exp \left[-i 2 \pi\left(x \frac{\alpha}{\lambda}+y \frac{\beta}{\lambda}\right)\right] d x d y
$$

Thus, by substituting $\frac{\alpha}{\lambda}$ with $f_{x}$ and $\frac{\beta}{\lambda}$ with $f_{y}$, the equation for the ASP of $U(x, y ; z)$ can be written as:

$$
U(x, y ; z)=\iint_{-\infty}^{+\infty} A\left(f_{x}, f_{y} ; z\right) \exp \left[i 2 \pi\left(x f_{x}+y f_{y}\right)\right] d f_{x} d f_{y}
$$

Besides, if $\sqrt{f_{x}^{2}+f_{y}^{2}}<\frac{1}{\lambda}$ condition is satisfied, $A\left(f_{x}, f_{y} ; z\right)$ will be expressed by [20]:

$$
A\left(f_{x}, f_{y} ; z\right)=A\left(f_{x}, f_{y} ; 0\right) \times \exp \left(i 2 \pi \frac{z}{\lambda} \sqrt{1-\left(\lambda f_{x}\right)^{2}-\left(\lambda f_{y}\right)^{2}}\right)
$$

with $\exp \left(\frac{2 i \pi z}{\lambda} \sqrt{1-\left(\lambda f_{x}\right)^{2}-\left(\lambda f_{y}\right)^{2}}\right)$ corresponding to a propagation kernel.

It is important to note that the angular spectrum picture has the advantage of being more intuitive and free from some of the subtle difficulties of boundary conditions. It also leads to a more robust and trouble-free numerical calculations of diffraction, since no approximation is necessary. However, it has the drawback of calculating only the light propagation close to the source. Notably, some artifacts appear when computing the Angular Spectrum Method (ASM) at long distances numerically. This limitation is due to the aliasing problem caused by the representation of the complex wave field $(U)$ as the superposition of an infinite number of plane waves. 


\subsubsection{Phase Shifting Digital Holography (PSDH)}

The concept of phase shifting interferometry has been introduced by Yamaguchi et al [3] to improve reconstruction quality in digital holography. Compared to single in-line/off-axis holograms, phase-shifting holography targets to enhance the reconstruction quality in $\mathrm{DH}$ by recording more information, at least three holograms associated to three reference beams with different phase shifts. In the simplest form of PSDH, the initial phase $\phi$ is zero and changes by $\frac{\pi}{2}$ at each step [21]. Doing so, the intensity of the interference patterns presented in Eq. 4 is updated as follows:

$$
I_{H}(x, y, \phi)=\left|U_{O}(x, y)+U_{R}(x, y, \phi)\right|^{2} \quad \phi \in\left\{0, \frac{\pi}{2}, \pi\right\}
$$

The complex amplitude of the object wavefront at the hologram plane is then calculated using the three phase-shifted interference patterns:

$$
\begin{aligned}
\hat{U}_{O}(x, y)= & \frac{1-j}{4 U_{R}^{*}}\left\{I_{H}(x, y, 0)-I_{H}\left(x, y, \frac{\pi}{2}\right)\right. \\
& \left.+i\left[I_{H}\left(x, y, \frac{\pi}{2}\right)-I_{H}(x, y, \pi)\right]\right\}
\end{aligned}
$$

One of the main advantages of the phase-shifting set-up is the elimination of the twin and the zero order images, as both these images degrade the reconstruction quality. However, this kind of set-up requires several captures for the same object and the phase-shifts must be highly accurate. Since the reconstruction quality from one single (in-line/off-axis) hologram is badly influenced by the bothering images due to the lost of phase information, in the rest of this paper we only consider the phase-shifting digital holograms.

\subsection{Representation Formats of Computer Generated Phase-Shifting} Holograms

According to the PSDH principle, and considering Eq. 17, the various formatting options of the complex object wave at the hologram plane are described in the following:

\subsubsection{Intensity based representation}

The first option is to obviously represent the intensity of the three phaseshifting holograms in Eq. 17; but this induces the coding of three sets of data and may hinder the coding efficiency. While still representing intensities, an other alternative consists in defining two sets of difference data: D1 and D2, derived from Eq. 17, and expressed as follows:

$$
\begin{aligned}
& D 1(x, y)=I_{H}(x, y, 0)-I_{H}\left(x, y, \frac{\pi}{2}\right) \\
& D 2(x, y)=I_{H}\left(x, y, \frac{\pi}{2}\right)-I_{H}(x, y, \pi)
\end{aligned}
$$


This type of representation is named shifted distance data since it is associated to the difference of phase-shifted holograms.

\subsubsection{Complex object field based representation}

The two expressions of complex number, defined in cartesian coordinate system and polar coordinate system lead to two representations of the interference pattern.

Real/Imaginary data approach: in this case, the complex field obtained in Eq. 17 is expressed as:

$$
\hat{U}_{O}(x, y)=\operatorname{Re}\left(\hat{U}_{O}(x, y)\right)+i \operatorname{Img}\left(\hat{U}_{O}(x, y)\right)
$$

with Re and Img being the real and imaginary parts of the complex object field at the hologram plane, respectively.

Amplitude/Phase data approach: another way for expressing the complex field is by the polar coordinate system, using the Euler's formula as:

$$
\hat{U}_{O}(x, y)=\hat{A}_{O}(x, y) \exp \left(i \hat{\omega}_{O}(x, y)\right)
$$

where $\hat{A}_{O}(x, y)$ and $\hat{\omega}_{O}(x, y)$ can be calculated using the real and imaginary parts defined in the coordinate system.

It is important to emphasis that the use of conventional image or video coding solutions on digital holograms, necessitates a scaling transformation to obtain components represented by 8 bit integers as imposed by the input type of the compression algorithm.

\section{State of the art on lossy compression of PSDH}

To compactly encode holographic data so that it becomes adequate to transmit over existing networks, some hologram compression techniques have emerged in recent years. In the case of phase-shifting digital holograms, three main compression strategies are used: interference patterns compression, camera-plane compression and compression at the reconstruction plane. By using the first category each of the three recorded interference patterns $I_{H}(:,:, \phi)$ is individually compressed. Since they are real-valued grayscale images, these interferograms can be compressed using conventional image coding methods. Despite the fact that they were designed for real-world images and not for interference patterns, it has been demonstrated $[36,37]$ that the fringe information of the interference patterns can be effectively preserved by these compression techniques. The second alternative consists in applying camera plane compression on phase-shifting interferometry (PSI) digital hologram $U(x, y)$, calculated according to Eq. 17. In the work presented in [23], Mills and Yamaguchi proposed to perform scalar quantization with 4 bit-depth (which corresponds to a compression ratio equals to 16), on real-imaginary components of the complex 
object field. To improve the compression performance, in terms of the reconstruction quality, Naughton et al. improved the quantization of real-imaginary components with a bit packing operation for real-time networking application [24]. Naughton et al. have extended their work by using a companding histogram approach for nonuniform quantization $[22,25]$. The quantizer step size selection is performed according to the distribution of the hologram components in the histogram. Another alternative, based on scalar quantization has been proposed in [26]. The main idea consists in coding digital holograms by applying a multiple description coding (MDC) method on amplitude-phase components using maximum-a-posteriori optimization approach.

Comparative study of scalar and vector quantization on different phaseshifting digital holographic data representations has been performed by Xing et al. [11]. According to this study, the authors have showed that both quantization methods allow to achieve a compression ratio of about 16 while still maintaining the visual quality of the compressed hologram at an acceptable level. The authors also stated that the vector quantization outperforms scalar quantization by an average of $1 \mathrm{~dB}$ for the amplitude/phase format and $3 \mathrm{~dB}$ for the shifted distance format.

On the other hand, various transform-based coding approaches have been investigated to compress PSI holograms. To exploit the redundancies in two sets of holographic information, obtained by PSDH setup, Xing et al. [21] developed a JPEG 2000 based compression method. They proposed to jointly encode the phase-shifting holographic data using a wavelet decomposition based on separable vector lifting scheme SEP-VLS. After that, they proposed a Non Separable (NS) extension of SEP-VLS, denoted by NS-VLS, to simultaneously exploit the correlation between the shifted distances, as well as their twodimensional isotropic characteristics [40]. After examining the experimental results, it has been concluded that Xing et al.'s compression method outperforms the reference coding methods, achieving a gain of about $2 \mathrm{~dB}$. The analysis also demonstrated that strangely enough, the "Standard Coding" method, which encodes a reference and a residual image using JPEG2000 in its lossy mode, has lower performance than the "Independent Coding" scheme, where the inputs are separately encoded by also using lossy mode of JPEG2000 compression. This seems to reveal that the existing residual coding methods are not able to efficiently exploit the inter-image redundancy for the tested hologram formats. To obtain further improvement in compression performance, Xing et al. used HEVC compression algorithm to encode PSI holograms based on their vector lifting scheme [13]. According to the experimental results, it has been demonstrated that the separable VLS decomposition based HEVC scheme slightly outperforms the HEVC intra mode but the HEVC inter mode still yields the best performance.

The hologram at the object plane (or reconstruction domain) looks like an image and might be much better compressible with conventional codecs. Thus, when the complex wavefield is back-propagated to the reconstruction plane before compression, apparent structure at the reconstruction plane is expected to increase the effectiveness of the lossless coder and leads to over- 
all higher compression performance. Consequently, some algorithms have been developed for the compression of phase shifting digital holograms, at the object plane. The first one has been proposed by Darakis and Soraghan [36] by investigated uniform scalar quantization followed by lossless coding. Later, the authors demonstrated that the Fresnelet transform of phase-shifting holograms in combination with SPIHT or uniform quantization can be used effectively for the compression of such holographic data [28]. Thereafter, Kari et al. [30] have proven that Fresnelets are not well localized in space and frequency and cannot form a good basis for compactly representing holograms for view dependent systems. In the counterpart, they have argued that Gabor wavelets are very well suited for preserving the local spatial frequencies of holograms [31]. To put their findings into practice, they developed two view-based compression techniques, using Fresnelets and Gabor wavelets, respectively. By comparing both compression methods on the basis of space-frequency localization, it has been experimentally confirmed that Gabor wavelets are able to suppress the unwanted orders created in the reconstruction process. However, their use entails a very high computational cost at the encoding stage. An other disadvantage that hamper the use of Gabor wavelets is the failure of the admissibility condition which ensures reversibility and unequal number of the sinusoids for various frequencies that causes an inefficient pruning of coefficients for viewdependent reconstruction [32]. To overcome these limitations, Rhammad et al. developed a color hologram compression method based on scalar and vector matching pursuit using an overcomplete Gabor's dictionary [45]. Experimental results revealed that Rhammad et al.'s method keeps consistent rate/distorsion performance and visual quality independently of the observer focus and point of view. They also demonstrated that scalar matching pursuit is more efficient for holograms having a low correlation between their channels, whereas vector matching pursuit performs better for holograms with strong correlation.

Recently, Peixeiro et al. [15] benchmarked the main available image coding standard solutions for Phase-Shifting Digital Holograms using interference patterns and PSI hologram representations. Comparisons between JPEG [35], JPEG 2000 [33], H.264 AVC [44] and HEVC [46] have been made in terms compression performance. The authors have demonstrated that the HEVC Intra coding mode is the best standard coding solution. Based on this observation, Marco et al. [16] have lately used HEVC intra main coding profile to analyse the coding performance of the different complex field representations of CGHs, in both the hologram plane and the object plane. This study reveals two significant conclusions. The first one is that the real-imaginary data is coded with higher efficiency than the amplitude-phase data. The second is that the object plane compression is a very efficient model that outperforms the compression on the hologram plane. Even if transformation from the object plane to another plane would be required in the case of holographic displays, or other rendering applications such as extended depth of focus or multiple perspectives, the added transformation complexity is negligible when compared with the hologram plane coding and display rendering computational complexity. 
3.1 Limitations and drawbacks in the state-of-the-art

Lossy compression is absolutely necessary to bridging the gap between PSI hologram coding and the application world of 3D graphics. However, due to the specific nature of PSI holographic data, it is extremely hard to understand the current status quo of their compression methods. No paper till date has been able to offer comprehensive study on the compression efficiency for true $3 \mathrm{D}$ immersive environments. This is mainly due to the fact that the way holographic data is generated, reconstructed, and represented has a great impact on how well it will be compressed. Most of the compression works, discussed above, are not strictly comparable due to the complicated system of PSDH. Typically, the fringes appearing on the holograms can be very different according to setup conditions such as the object numbers, the recording distance, the recording condition,...In most cases, the researchers in the field of PSDH compression, used their private data to perform the experimental evaluations. Although, the type of the hologram should not be a deciding factor on the compression, since such restriction will put a strong constraint on the encoding process of holograms. Furthermore, there is no standard metrics, specific for holograms, for assessing the compression performance or the reconstruction accuracy.

Some work have tackled the problem of hologram compression by using compact wavelet representation. When doing so, it is immediately clear that wavelets are not really suited to hologram coding in their classical form. Indeed, conventional wavelets are well-suited for approximating data with sharp discontinuities. This is not the case for holograms where the information that is localized in the 3D scene is spread out in the entire hologram, under the form of very rapidly oscillating patterns. Some new techniques have been developed to handle this type of information by using Fresnelets and Gabor transforms which can benefit from the diffraction property. But their practical use in sophisticated schemes taking into consideration the object based compression and complexity-rate-distortion tradeoff has not yet been investigated. Regarding the stat-of-the-art in lossy compression for PSDH data, we mention that it is utmost important to take into consideration the computational cost of the different encoding paradigms. For all the aforementioned coding solutions, it was not possible to provide the complexity assessment because the results are missing. Obviously, one should carefully select the proper design methodology depending on the required functionality of the codec that is to be implemented. Given the fact that backward compatibility with legacy JPEG is crucial in the context of image sharing applications, it may be interesting to investigate JPEG-based coding approaches in the field of PSDH compression. Furthermore, according to Darakis and Soraghan [38] findings and those obtained by Marco et al.'s [16], the encoding of holographic information in the object plane can be more convenient in numerous interesting applications. 


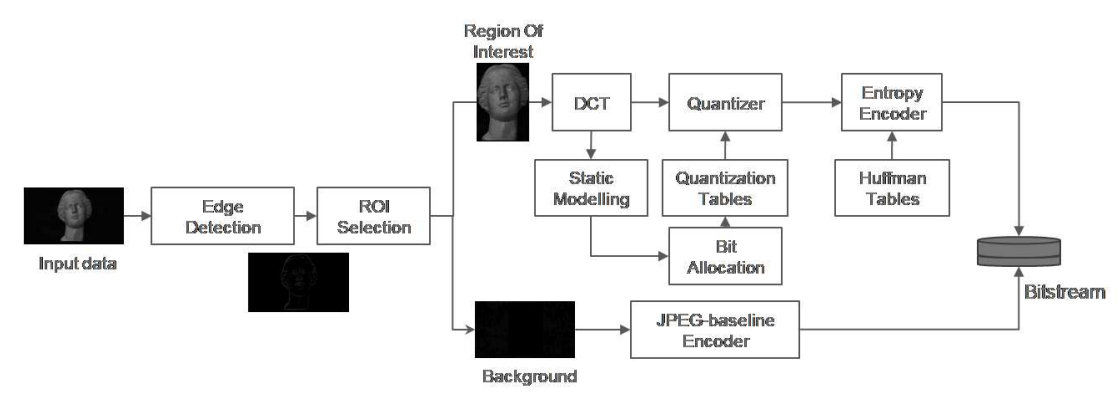

Fig. 2: Block diagram of the proposed object based coding method.

\section{Description of the Proposed Coding Algorithm}

In this work, we present a coding method for phase-shifting digital holograms that consists in integrating a region-of-interest-based bit allocation in JPEG encoder. The target goal is to take advantage of the simplicity of baseline JPEG encoder and the higher efficiency of optimised bit rate allocation. Our approach is supported by the fact that hologram data usually consists of a region representing the object under investigation on an often noisy background of no interest. It seems very natural therefore to process such data in an object-based framework by assigning high priority to the semantically relevant object region and lower priority to the background. More specifically, the relevant object is selectively encoded by using a near optimal JPEG based algorithm, that offers the best rate/distorsion tradeoff in terms of Lagrangian cost. In doing so, a pre-segmentation procedure is required to identify and segment the ROI before encoding. The block diagram of the proposed coding scheme is illustrated by Fig. 2 .

It is worth mentioning that our coding method is adapted to PSDH data represented in object plane, but it can be easily extended for use in hologram plane on the condition of applying a scaling that converts hologram samples, with floating point values, to 8 bits integers.

\subsection{Pre-segmentation}

The pre-segmentation stage is divided in two steps. The first one involves performing an edge detection to identify the boundaries of homogeneous regions in the hologram data based on classical image properties such as intensity and texture. In our work, we propose to use the Canny algorithm [48] which has the advantage to be simple while providing precise and reliable detection.

In the second step, the ROI, which is taken as a rectangular area, is selected automatically according to the detected edges. Specifically, the contours resulting from Canny detection algorithm are first represented by a vector of 
points. Each point is identified by its spatial coordinates $x$ and $y$ in the image. Among the recovered edge points, the four extremity points $\mathbf{p}_{i}(x, y)$, $i \in 1, \ldots, 4$, of the rectangular ROI to be cropped, are determined by selecting the minimum and maximum values of $x$ and $y$ coordinates. Let's designate by $x_{\min }, y_{\min }, x_{\max }, y_{\max }$, the minimum and the maximum coordinate values, respectively. The four extremity points are identified as follows:

$$
\begin{aligned}
& \mathbf{p}_{1}(x, y): \text { with } x=x_{\min } \text { and } y=y_{\min } \\
& \mathbf{p}_{2}(x, y): \text { with } x=x_{\min } \text { and } y=y_{\max } \\
& \mathbf{p}_{3}(x, y): \text { with } x=x_{\max } \text { and } y=y_{\min } \\
& \mathbf{p}_{4}(x, y): \text { with } x=x_{\max } \text { and } y=y_{\max }
\end{aligned}
$$

To increase the precision of the ROI selection when Canny's filter [48] fails to accurately detect the contours, we suggest to slightly enlarge the ROI. By this way, we guarantee that the missing parts of the relevant object will be recovered. Thereby, we defined two scaling parameters $\mu_{w}$ and $\mu_{h}$ that vary with hologram image width $W$ and height $H$, in addition to a fixed integer parameter $\beta$. Notably,

$$
\mu_{w}=\frac{W}{\beta} \text { and } \mu_{h}=\frac{H}{\beta}
$$

In such a manner, the key points coordinates will be shifted as follows:

$$
\begin{aligned}
x_{\min } & =x_{\min }-\mu_{w}, \\
x_{\max } & =x_{\max }+\mu_{w}, \\
y_{\min } & =y_{\min }-\mu_{h}, \\
y_{\max } & =y_{\max }+\mu_{h} .
\end{aligned}
$$

Prior to the compression purpose, a binary masking is applied to the segmented hologram image to separate the ROI from the image background. In doing so, the ROI will be encoded using a a near optimal bit allocation based on Lagrangian cost. on the other side, the background region is compressed, at lower compression efficiency, using the baseline version of JPEG algorithm. In the next subsection we briefly describe JPEG-OPT coding scheme that has been chosen to encode the ROI.

\subsection{Bit allocation approach}

In this section, we describe the bit allocation approach that consists of nearoptimal rate control in DCT-based compression while ensuring backward compatibility with legacy JPEG decoder.

Assuming that the ROI, of size $h \times w$, is a set $I$ of $\frac{h \times w}{64}$ blocks. After applying the DCT to each $8 \times 8$ of the ROI, the set of spatial frequencies, in each DCT block is ordered into a 1-D array of 64 coefficients using the zig-zag 
scan. A DCT coefficient with coordinates $(k, l),(k, l=1, \ldots, 8)$, is then indexed by $n=8 \times k+l$. The $n^{\text {th }}$ coefficient belonging to the DCT block $C^{b}$ is then denoted by $C[n]^{b}$, where $n$ is the coefficient location and $b$ is the block index.

Before passing through the Huffman entropy coder, the quantized DCT coefficients undergo run-length coding, which combines zero DCT indices from different frequency bins into one symbol.

The JPEG syntax leaves the selection of the quantization table to the encoder. Unlike baseline JPEG encoder, the proposed encoder uses a near optimal bit allocation procedure to determine an efficient image-adaptive quantization table. Particularly, the trade-off between total bit rate $R$, and total distortion $D$, is balanced by minimizing the Lagrange cost function $J(\lambda)$ with $\lambda$ being the Lagrange multiplier. By other means, the near optimal quantization table $Q^{*}$ is the one that minimizes $J(\lambda)=D(Q)+\lambda R(Q)$.

According to Ratnakar and Livny [18] studies, the zero run-length coding, hinders the use of classical optimal bit allocation methods. An alternative strategy is to propose a sub-optimal solution that uses histograms of the probability distribution of the coefficients over all the DCT blocks.

Based on Parseval theorem, the total distortion that affects an input block $I^{b}$, from the original ROI, can be replaced by the sum of the coefficient distortions in its corresponding DCT coefficient block $C^{b}$. In addition, the bit rate can be approximated as the sum of the entropies, over the 64 frequency emplacements, of the quantized DCT coefficients over all the the blocks in the ROI. Given a quantization table $Q$, let $D(Q)$ and $R(Q)$ represent the Mean square error (MSE) and the estimated bit rate (in bits per sample), respectively, resulting from compressing the ROI in a PSDH using the quantization table $Q$. For a given quantizer step size $q, D[n][q]$, the contribution to the total distortion at the $n^{\text {th }}$ frequency emplacement is given by:

$$
D[n][q]=\frac{1}{64} \operatorname{Mean}\left(\left(C[n]-\operatorname{Round}\left(\frac{C[n]}{q}\right)\right)^{2}\right),
$$

where the "Mean" is taken over all the $8 \times 8$ blocks in the quantized and DCT domain. Similarly, the rate contribution, $R[n]\left[q_{n}\right]$, at the $n^{\text {th }}$ frequency localisation is expressed by:

$$
R[n][q]=\operatorname{Entropy}\left(\operatorname{Round}\left(\frac{C[n]}{q}\right)\right) .
$$

Note that $R[n][q]$ corresponds to the entropy of the quantized DCT coefficients at the $n^{\text {th }}$ frequency position. It is calculated using the distribution histogram at the same frequency position.

For a specific quantization table $Q$, the estimated total bit rate is given by:

$$
R(Q)=\sum_{n=0}^{63} R[n][Q[n]],
$$


whereas the total distortion is estimated by:

$$
D(Q)=\sum_{n=0}^{63} D[n][Q[n]]
$$

Giving $Q_{\min }$ and $Q_{\max }$ the quantization tables that, respectively, represent the minimum and maximum values tolerable for each quantization table entry, such that, $Q_{\min }[n] \leq Q[n] \leq Q_{\max }[n]$, the rate-distortion optimisation scheme builds tables of $D[n][q]$ and $R[n][q]$, for each quantizer step size $q=Q[n]$ in the predefined operating range.

The rate-distortion optimisation problem is then formulated by the minimization of Lagrangian cost function. By using Eqs 23 and 25, the Lagrangian cost function, $J(\lambda)$, is formulated by:

$$
J(\lambda)=\sum_{n=0}^{63}(D[n][Q[n]]+\lambda R[n][Q[n]]) .
$$

For each frequency localization $n$, a subset of the operating points $(R[n][],. D[n][]$. are pruned and sorted such that $R[n][$.$] is strictly decreasing, whereas D[n][$. is strictly increasing. The Graham scan algorithm [43] is used to get the (lower half) convex hull of the $(R[n][],. D[n][]$.$) points. The Graham algorithm begins$ at the left-most point (which is certainly on the convex hull), and then preserves points in increasing order of distortion. If the line segment from the previous point to the new point is a right turn with respect to the last such line segment, in that case the previous point is eliminated from the hull. The eliminations continue until a left turn is possible. Let $h_{n}$ designate the remaining points on the hull and $\left\{q_{n}(1), \ldots, q_{n}\left(h_{n}\right)\right\}$ the associated quantizer values. The slopes of the rate-distortion curve for the $n^{\text {th }}$ frequency position at these $h_{n}$ points are expressed by:

$$
\lambda_{n}(k)=\frac{R_{n}\left(q_{n}(k)\right)-R_{n}\left(q_{n}(k+1)\right)}{D_{n}\left(q_{n}(k+1)\right)-D_{n}\left(q_{n}(k)\right)},
$$

for $k=1,2, \ldots, h_{n}-1$ and $\lambda_{n}\left(h_{n}\right)$ is set to zero. Hence, $\lambda_{n}(k)>\lambda_{n}(k+1)$, for $k=1,2, \ldots, h_{n}-1$. Notably, for any given $\lambda>0$, the Lagrangian cost function, $J(\lambda)=D[n][q]+\lambda R[n][q]$ is minimized for $q=q_{n}(k)$, with $k$ being the least index for which $\lambda>\lambda_{n}(k)$. More generally, for any given $\lambda>0$, the 64 binary searches can be used to find the quantizer values, belonging to the operating range, such that $R(Q)+\lambda D(Q)=\sum_{n=0}^{63} D[n][Q[n]]+\lambda R[n][Q[n]]$ is minimized. If a target bit rate $R^{*}$ is considered, the bisection method is used to easily search for a $\lambda$ that would satisfy the target up with respect to the fixed tolerance.

At a high level understanding, the bit allocation algorithm can be summarized as following: 


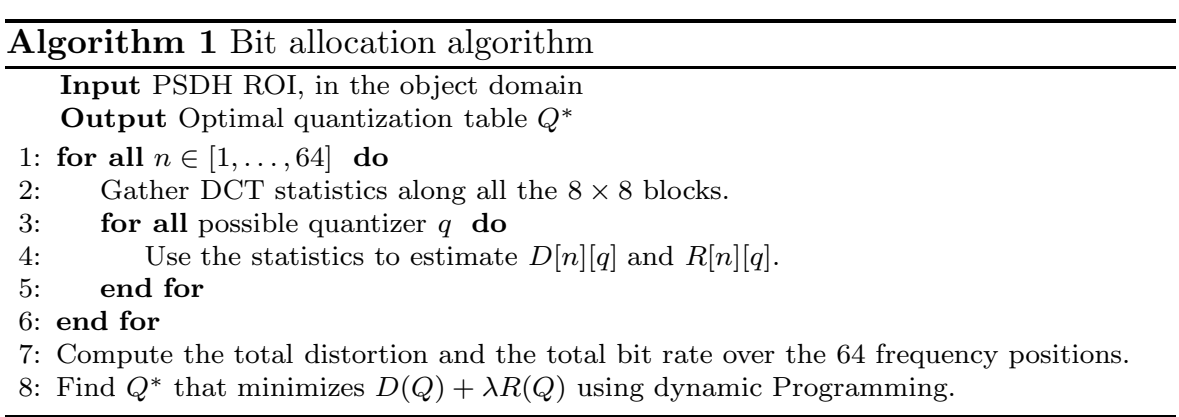

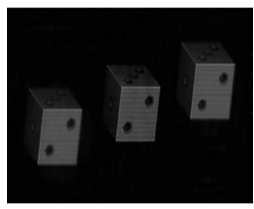

2D Multi

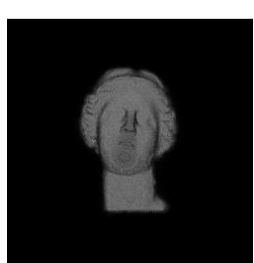

Venus8KS

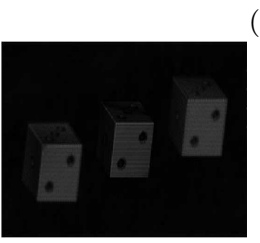

3D Multi

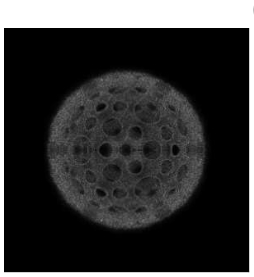

Ball8KS

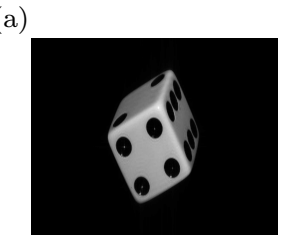

2D Dice

(b)

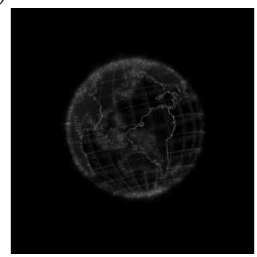

Earth8KS

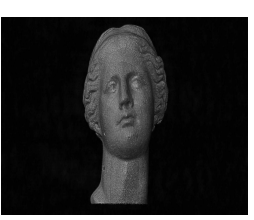

$3 \mathrm{D}$ Venus

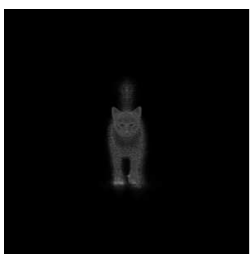

Cat8KS

Fig. 3: Synthetic holograms selected from Interfere-I (a) and Interfere-II (b) databases.

\section{Performance evaluation}

Experiments have been conducted to evaluate the proposed coding scheme on Phase-shifting digital holograms. Based on Darakis et al. [38] and Marco et al. [16]findings, we have chosen to perform our compression method in the object plane, while considering only the amplitude of the reconstructed object. Since phase information is eliminated only a particular view is represented. But the reconstruction of other object views remain possible by using specific transformation from the object plane to other object configurations. We also emphasize that our method can be applied in the hologram plane with respect to a scaling pre-processing that reduces the bit depth to 8 bits integers. 
Table 1: Parameters of CGH generation setups.

\begin{tabular}{lcccc}
\hline & $\begin{array}{c}\text { Resolution } \\
(\text { pixel})\end{array}$ & $\begin{array}{c}\text { Pixel } \\
\text { pitch }(\mu \mathrm{m})\end{array}$ & $\begin{array}{c}\text { Reconstruction } \\
\text { distance }(\mathrm{m})\end{array}$ & $\begin{array}{c}\text { Wavelength } \\
(\mathrm{nm})\end{array}$ \\
\hline 2D Dice & $1920 \times 1080$ & 8 & 0.90 & 632.8 \\
2D Multi & $1920 \times 1080$ & 8 & 0.90 & 632.8 \\
3D Venus & $1920 \times 1080$ & 8 & 0.50 & 632.8 \\
3D Multi & $1920 \times 1080$ & 8 & 0.10 & 632.8 \\
\hline Venus8KS & $8192 \times 8192$ & 1 & 0.0129 & 633 \\
Earth8KS & $8192 \times 8192$ & 1 & 0.0118 & 633 \\
Cat8KS & $8192 \times 8192$ & 1 & 0.0142 & 633 \\
Ball8KS & $8192 \times 8192$ & 1 & 0.0125 & 633 \\
\hline
\end{tabular}

\subsection{Experimental setup}

Our simulations have been carried out using PSDH data, selected from InterfereI (2D-Venus, 2D-Multi, 3D-Multi and 3D-Venus) and Interfere-II (Venus8KS, Ball8KS, Earth8KS and Cat8KS) databases. The characteristics of the considered holograms, depicted in Fig.3, are summarized in Table.1.

The Interfere databases was made available, by Prof. Peter Schelkens from ETRO-VUB, at http://www.erc-interfere.eu.

- Interfere-I database: consists of 5 virtual objects. The holograms were computer generated using a light wave propagation strategy that is able to handle occlusions and the Angular Spectrum Propagation model.

- Interfere-II database: contains 6 diffuse and 6 specular CGHs generated from 3D point clouds. The diffuse CGHs were generated by assigning a random phase intensity per point, whereas specular CGHs have only real intensity per point.

The reconstructed object, to be compressed, were obtained by using the physical focus depth as reconstruction distance. It is worth mentioning that the reconstruction distance parameter can highly influences the compression performance if it is not kept within the physical depth limits of the scene. In that case, the best reconstruction distance can be calculated using an auto focus function.

To assess the effectiveness of our coding method we conducted a series of tests by measuring the quality of the decoded hologram data as a function of the attained bitrates. The quality distortion has been measured in both the object and the hologram plane. Since we performed the compression tests on the object plane, an additional processing step, which consists of inverse reconstruction, has been applied to the decoded data to compare the original hologram, represented with Real-Imaginary representation format to the decoded one using the same representation format. In order not to deviate from previous work, the NRMS has been used for the quality assessment in the hologram domain, whereas the PSNR has been used to evaluate the quality in the object domain.

Initially, we start by analyzing the impact of our compression method on the reconstruction quality while considering the whole depth range of the 
Table 2: Compression performance evaluation (in bitrate (bps), PSNR (dB) and reconstruction distance) for Venus $8 \mathrm{KS}$ and Cat8KS.

\begin{tabular}{llllll}
\hline \multirow{2}{*}{ Model } & \multirow{2}{*}{$\begin{array}{l}\text { Depth range } \\
(\mathrm{m})\end{array}$} & \multicolumn{2}{c}{$\begin{array}{l}\text { Reconstruction } \\
\text { distance }(\mathrm{m})\end{array}$} & \multicolumn{3}{c}{ Bitrate (bps) } \\
\cline { 4 - 6 } & & 0.1 & 0.3 & 0.5 \\
\hline \multirow{3}{*}{ Venus8KS } & \multirow{3}{*}{$0.0128-0.0171$} & 0.0150 & 40.36 & 49.65 & 58.62 \\
& & 0.0140 & 40.37 & 49.65 & 58.64 \\
& & 0.0160 & 40.38 & 49.66 & 49.68 \\
& & 0.0170 & 40.39 & 49.66 & 58.65 \\
Cat8KS & \multirow{3}{*}{$0.0140-0.0207$} & 0.0157 & 38.06 & 44.65 & 52.68 \\
& & 0.0172 & 38.07 & 44.65 & 52.68 \\
& & 0.0187 & 38.08 & 44.65 & 52.68 \\
& & 0.0202 & 38.08 & 44.67 & 52.69 \\
& & & 38.08 & 44.66 & 52.69 \\
\hline
\end{tabular}

holographic data. Secondly, we evaluate the performance of our compression method and compare it with those obtained by the reference methods: JPEG [35], JPEG 2000 [33], QT-L [47], SPIHT [29], JPEG and HEVC [46]. It is worth noting that J2K (JPEG 2000), QT-L and SPIHT are wavelet-based compression techniques. JPEG 2000 and QT-L codecs exploit the intra-band dependencies between wavelet coefficients, whereas SPIHT codec exploits the inter-band dependencies between the wavelet sub-bands and employs a zerotree structure to drop the insignificant coefficients. To offer a fair comparison, the tested wavelet-based encoders used the following set of parameters: irreversible $(9,7)$ filter-bank; 3 levels of dyadic wavelet decomposition; and a single layer not considering the progressive decoding mode.

For JPEG encoder, the rate-distortion results were obtained by varying the quality parameter until the desired target bitrate is reached. Moving on to the experiment for HEVC encoder, in our simulations we used the HEVC Test Model 16.9. Note that YUV format is the main raw video format used in video coding softwares. Thus, before HEVC encoding, the reconstructed digital holograms were converted to YUV 4:2:0 format to satisfy the input format supported by the Main Still Picture profile in the reference software. Since dynamic data is not considered, we only enabled the HEVC Intra coding mode. The latter adopts a similar quantization step value for all frequencies. Thus, each holographic data (in the objevt plane) is coded using six different Intra quantization steps, taking the values $22,27,32,37,42$ and 47 , to consider various levels of decoded quality.

\subsection{Compression effect on different depth reconstructions}

As holograms encompasses three dimensional information we investigate the compression impact on different distance reconstructions. In this way we evaluate the compression performance of our method for the whole depth range of the holographic data. Specifically, simulations have been conducted by performing final reconstructions (from the compressed data) by selecting various 
(a)

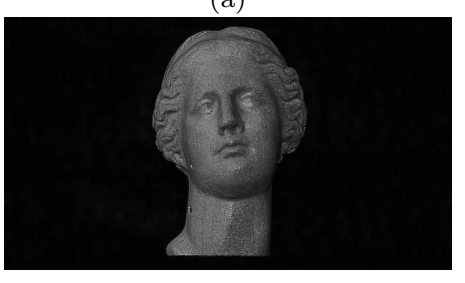

(c)

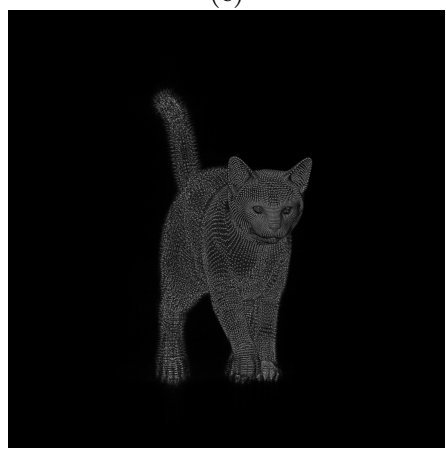

(b)

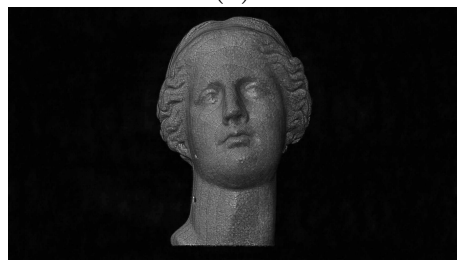

(d)

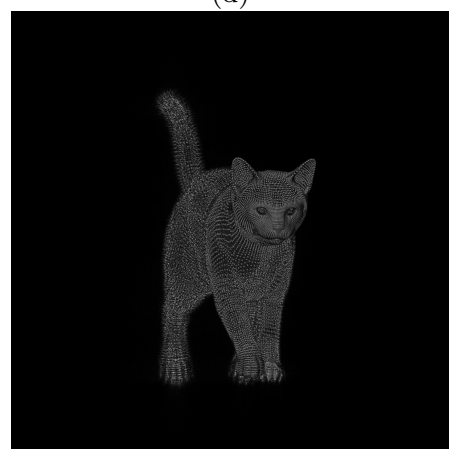

Fig. 4: Visual comparison of Venus8KS and Cat8KS decoded at $0.3 \mathrm{bps}$ with our method and using different depth reconstructions " $z$ ": (a) $z=0.0129$ and (b) $z=0.0170$ for Venus $8 \mathrm{KS}$; (c) $z=0.0142$ and (d) $z=0.0202$ for Cat8KS.

Table 3: Compression performance evaluation (in bitrate (bps) and PSNR (dB)) when using the reconstructed holograms collected from Interfere-I database.

\begin{tabular}{|c|c|c|c|c|c|c|c|}
\hline \multirow{2}{*}{ Model } & \multirow{2}{*}{$\begin{array}{l}\text { Bitrate } \\
\text { (bps) }\end{array}$} & \multicolumn{6}{|c|}{ PSNR (dB) } \\
\hline & & $\begin{array}{l}\text { Our } \\
\text { method }\end{array}$ & JPEG & $\mathrm{J} 2 \mathrm{~K}$ & QT-L & SPIHT & HEVC \\
\hline \multirow{3}{*}{ 2D Dice } & 0.2 & 57.96 & 56.13 & 57.10 & 57.15 & 58.32 & 59.1 \\
\hline & 0.4 & 62.18 & 60.27 & 61.76 & 61.74 & 63.35 & 63.48 \\
\hline & 0.6 & 65.75 & 64.05 & 65.12 & 64.98 & 65.89 & 65.91 \\
\hline \multirow{5}{*}{ 2D Multi } & 0.2 & 31.3 & 29.96 & 31.03 & 31.32 & 33.14 & 34.63 \\
\hline & 0.4 & 33.48 & 30.17 & 32.88 & 33.18 & 35.65 & 36.85 \\
\hline & 0.6 & 36.89 & 32.96 & 35.13 & 35.56 & 37.85 & 39.12 \\
\hline & 0.8 & 39.4 & 36.96 & 38.42 & 38.52 & 40.56 & 41.23 \\
\hline & 1 & 44.18 & 41.32 & 43.89 & 43.21 & 44.11 & 44.36 \\
\hline \multirow{5}{*}{ 3D Venus } & 0.2 & 34.23 & 33.73 & 34.01 & 34.06 & 34.76 & 34.91 \\
\hline & 0.4 & 37.78 & 36.99 & 37.42 & 37.49 & 38.12 & 38.36 \\
\hline & 0.6 & 41.61 & 38.93 & 41.13 & 41.20 & 41.75 & 41.81 \\
\hline & 0.8 & 44.34 & 40.42 & 44.09 & 44.02 & 44.41 & 44.45 \\
\hline & 1 & 46.68 & 42.16 & 46.32 & 46.11 & 46.62 & 46.66 \\
\hline \multirow{5}{*}{ 3D Multi } & 0.2 & 30.15 & 28.63 & 29.66 & 29.75 & 31.26 & 32.24 \\
\hline & 0.4 & 33.56 & 31.62 & 32.95 & 33.05 & 34.84 & 35.68 \\
\hline & 0.6 & 35.62 & 34.78 & 35.65 & 35.48 & 37.65 & 39.24 \\
\hline & 0.8 & 37.65 & 35.16 & 36.78 & 36.52 & 38.86 & 40.12 \\
\hline & 1 & 40.12 & 38.69 & 39.68 & 39.14 & 40.68 & 42.33 \\
\hline Average & & 40.326 & 39.607 & 41.278 & 40.792 & 42.434 & 43.360 \\
\hline
\end{tabular}


Table 4: Compression performance evaluation (in bitrate (bps) and PSNR (dB)) when using the reconstructed holograms collected from Interfere-II database.

\begin{tabular}{|c|c|c|c|c|c|c|c|}
\hline \multirow{2}{*}{ Model } & \multirow{2}{*}{$\begin{array}{l}\text { Bitrate } \\
\text { (bps) }\end{array}$} & \multicolumn{6}{|c|}{ PSNR (dB) } \\
\hline & & $\begin{array}{l}\text { Our } \\
\text { method }\end{array}$ & JPEG & $\mathrm{J} 2 \mathrm{~K}$ & QT-L & SPIHT & HEVC \\
\hline \multirow{3}{*}{ Venus8KS } & 0.1 & 40.36 & 39.12 & 40.36 & 40.88 & 41.32 & 42.11 \\
\hline & 0.3 & 49.65 & 47.86 & 49.56 & 49.17 & 49.12 & 50.63 \\
\hline & 0.5 & 58.62 & 56.78 & 58.65 & 57.13 & 57.36 & 59.63 \\
\hline \multirow{3}{*}{ Cat8KS } & 0.1 & 38.06 & 36.11 & 37.85 & 38.63 & 40.36 & 42.98 \\
\hline & 0.3 & 44.65 & 41.52 & 43.65 & 44.56 & 48.63 & 49.68 \\
\hline & 0.5 & 52.68 & 50.23 & 52.10 & 52.13 & 57.63 & 58.94 \\
\hline \multirow{5}{*}{ Ball8ks } & 0.2 & 38.68 & 36.17 & 38.13 & 39.89 & 40.32 & 41.52 \\
\hline & 0.4 & 42.56 & 39.41 & 40.32 & 41.24 & 43.56 & 45.32 \\
\hline & 0.6 & 48.12 & 45.36 & 46.52 & 46.65 & 47.95 & 49.63 \\
\hline & 0.8 & 52.14 & 49.78 & 51.29 & 50.63 & 51.62 & 53.78 \\
\hline & 1 & 58.69 & 55.63 & 57.10 & 56.32 & 57.89 & 59.11 \\
\hline \multirow{6}{*}{ Earth8KS } & 0.1 & 37.89 & 36.12 & 37.88 & 38.96 & 40.16 & 40.12 \\
\hline & 0.2 & 45.59 & 43.11 & 46.13 & 46.56 & 47.12 & 47.96 \\
\hline & 0.4 & 54.78 & 52.36 & 53.16 & 52.62 & 54.16 & 55.96 \\
\hline & 0.5 & 52.68 & 50.23 & 52.10 & 52.13 & 57.63 & 58.94 \\
\hline & 0.2 & 38.68 & 36.17 & 38.13 & 39.89 & 40.32 & 41.52 \\
\hline & 0.4 & 42.56 & 39.41 & 40.32 & 41.24 & 43.56 & 45.32 \\
\hline Average & & 47.319 & 44.968 & 46.621 & 46.812 & 48.371 & 49.812 \\
\hline
\end{tabular}

depth planes from the depth range of the object. Table 2 shows the PSNR results when applying our codec to Venus8KS and Cat8KS models. The compression was performed on the reconstructed wave-front from the reconstruction distance provided in Table 1, whereas the final reconstruction (from the compressed data) has been performed by using 5 depth planes. The latter have been selected at regular intervals (from lower to upper limit) over the depth range of the $3 \mathrm{D}$ object.

From the results reported in Table 2, we can notice that for all the tested depth planes, our compression scheme produces very similar results over reconstruction quality. For instance, in the case of Venus $8 \mathrm{KS}$, when using the compressed data to perform the reconstruction at the reconstruction distances 0.0129 and 0.0170 , on average the PSNR decrease is about $0.03 \mathrm{~dB}$ for the three tested bitrates. These observations are in line with the visual evaluations of the compressed and reconstructed hologram. Figure 4 provides renderings of Venus8KS and Cat8KS, after being compressed at 0.3 bps and then reconstructed at the extreme distances within the depth range. Examining this figure, we deduct that the obtained reconstructions are indistinguishable. One may thus conclude that for the tested hologram models (collected from Interfere II database), the proposed object based compression method is depthindependent. 
Table 5: Compression performance evaluation (in bitrate (bps) and NRMS) when using the reconstructed holograms collected from Interfere-I database.

\begin{tabular}{|c|c|c|c|c|c|c|c|}
\hline \multirow{2}{*}{ Model } & \multirow{2}{*}{ bpp } & \multicolumn{6}{|c|}{ NRMS } \\
\hline & & $\begin{array}{l}\text { Our } \\
\text { method }\end{array}$ & JPEG & $\mathrm{J} 2 \mathrm{~K}$ & QT-L & SPIHT & HEVC \\
\hline \multirow{3}{*}{ 2D Dice } & 0.2 & 0.41 & 0.51 & 0.44 & 0.47 & 0.43 & 0.49 \\
\hline & 0.4 & 0.33 & 0.44 & 0.39 & 0.38 & 0.35 & 0.31 \\
\hline & 0.6 & 0.26 & 0.36 & 0.31 & 0.26 & 0.23 & 0.24 \\
\hline \multirow{5}{*}{ 2D Multi } & 0.2 & 1.64 & 1.75 & 1.67 & 1.68 & 1.67 & 1.65 \\
\hline & 0.4 & 1.43 & 1.59 & 1.45 & 1.52 & 1.54 & 1.49 \\
\hline & 0.6 & 1.28 & 1.37 & 1.32 & 1.29 & 1.31 & 1.26 \\
\hline & 0.8 & 1.19 & 1.29 & 1.21 & 1.22 & 1.27 & 1.13 \\
\hline & 1 & 1.15 & 1.26 & 1.17 & 1.17 & 1.23 & 1.08 \\
\hline \multirow{5}{*}{ 3D Venus } & 0.2 & 5.49 & 5.63 & 5.45 & 5.33 & 5.47 & 5.51 \\
\hline & 0.4 & 3.36 & 3.48 & 3.33 & 4.17 & 4.23 & 4.21 \\
\hline & 0.6 & 2.31 & 2.43 & 2.19 & 3.12 & 3.51 & 3.48 \\
\hline & 0.8 & 1.75 & 2.02 & 1.67 & 2.34 & 2.47 & 2.23 \\
\hline & 1 & 1.41 & 1.66 & 1.39 & 1.56 & 1.61 & 1.47 \\
\hline \multirow{5}{*}{ 3D Multi } & 0.2 & 2.21 & 2.37 & 2.24 & 2.27 & 2.24 & 2.21 \\
\hline & 0.4 & 2.14 & 2.25 & 1.17 & 2.11 & 2.17 & 2.13 \\
\hline & 0.6 & 1.86 & 2.13 & 1.93 & 1.88 & 1.97 & 1.93 \\
\hline & 0.8 & 1.71 & 2.01 & 1.84 & 1.73 & 1.76 & 1.74 \\
\hline & 1 & 1.59 & 1.87 & 1.72 & 1.54 & 1.51 & 1.49 \\
\hline Average & & 1.7511 & 1.912 & 1.771 & 1.886 & 1.942 & 1.893 \\
\hline
\end{tabular}

Table 6: Compression performance evaluation (in bitrate (bps) and NRMS) when using the reconstructed holograms collected from Interfere-II database.

\begin{tabular}{llllllll}
\hline \multirow{2}{*}{ Model } & bpp & \multicolumn{7}{c}{ NRMS } \\
\cline { 2 - 7 } & & $\begin{array}{l}\text { Our } \\
\text { method }\end{array}$ & JPEG & J2K & QT-L & SPIHT & HEVC \\
\hline \multirow{2}{*}{ Venus8KS } & 0.1 & 6.88 & 8.15 & 6.69 & 8.58 & 6.97 & 7.12 \\
& 0.3 & 6.51 & 7.92 & 6.53 & 8.11 & 6.72 & 7.03 \\
& 0.5 & 6.19 & 7.19 & 6.22 & 7.47 & 6.49 & 6.87 \\
\hline \multirow{2}{*}{ Cat8KS } & 0.1 & 5.72 & 7.35 & 6.16 & 6.89 & 5.17 & 5.88 \\
& 0.3 & 5.48 & 7.03 & 6.52 & 6.62 & 4.89 & 5.62 \\
& 0.5 & 5.34 & 6.78 & 5.71 & 6.17 & 4.61 & 5.31 \\
\hline \multirow{3}{*}{ Ball8ks } & 0.2 & 7.81 & 9.11 & 8.13 & 8.74 & 8.18 & 7.96 \\
& 0.4 & 7.66 & 8.83 & 7.82 & 8.55 & 7.78 & 7.52 \\
& 0.6 & 7.43 & 8.54 & 7.53 & 8.23 & 7.53 & 7.43 \\
& 0.8 & 6.97 & 8.17 & 7.21 & 8.07 & 7.21 & 7.27 \\
Earth8KS & 0.2 & 6.65 & 7.69 & 7.02 & 7.82 & 7.07 & 7.08 \\
\hline Average & 0.1 & 8.12 & 9.88 & 7.33 & 7.58 & 7.88 & 7.87 \\
\hline
\end{tabular}

5.3 Comparisons with other methods

For demonstrating the compression adequacy to hologram data, we also compared our method to the successful coding schemes: SPIHT, JPEG 2000 and QT-L, JPEG and HEVC. By examining the PSNR results reported in Table 
3 and Table 4 we notice that, the best results in terms of PSNR are obtained with HEVC followed by SPIHT. For low bitrate (between 0.1 and 0.5 ), we can see that JPEG-2000 and QT-L are very similar and that our method brings a slight improvement over these two wavelet based methods. At medium bitrates (from 0.6 to 1 ), the results provided by our method are very similar to those obtained by SPIHT and HEVC. Furthermore, the reported PSNRs results show that our method brings significant improvement over baseline JPEG encoder, which is evidently due to the use of sub-optimal bit allocation in conjunction with JPEG encoder.

In terms of visual quality, Figures 5 and 6 depict cropped regions from Earth8KS and Venus compressed with our method and HEVC. The visual testing reveals tha the proposed codec provides superior quality in the selected ROI. For both objects, the cropped region decoded by our method has a less noisy appearance, better preserved edges and sharper appearance. This is not surprising since our coding technique uses a nearly optimal rate/distortion tradeoff, to only construct specific quantization tables, in the ROI. While HEVC integrates a bit allocation algorithm that dynamically adjusts various encoder parameters, such as the motion parameters and the prediction mode, which are not profitable for static image coding.

Finally, we evaluate the Normalized Root-Mean-Square error (NRMS) in the complex hologram domain for low-to-medium bit rates. The simulation results reported in tables 5 and 6 show that the NRMS errors obtained using the proposed coding scheme are inferior to those obtained with the reference methods: JPEG [35], JPEG 2000 [33], QT-L [47], SPIHT [29], JPEG [35] and HEVC [46]. This is can be explained by the fact that the significant part of the PSDH image is coded with a very high quality by generating customized DCT quantization tables. The latter are selected according to a bit allocation approach that takes into account the rate/distortion tradeoff. Consequently, we can conclude that the proposed optimized coding scheme offers higher performance when considering the phase shifting holograms in the hologram domain.

\subsection{Comparative review}

To summarize, the results of the conducted experiments we can plainly discern that:

- In our method, the ROI position and shape do not undergo a significant change with the selected reconstruction distance.

- HEVC produces the best quality in terms of PSNR. But this is at the expense of extremely high computational complexity and coding time [49], which is not profitable for still image coding.

- SPIHT offers the highest compression performance, compared to the tested compression methods, with the exception of HEVC. According the our previous work [49], its has been demonstrated that SPIHT requires the lowest coding and decoding times among all the tested codecs. 
(a)

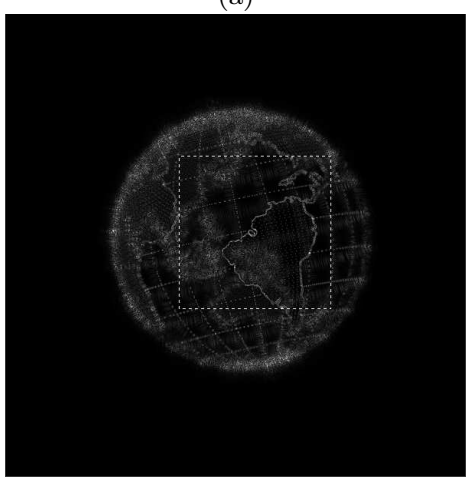

(c)

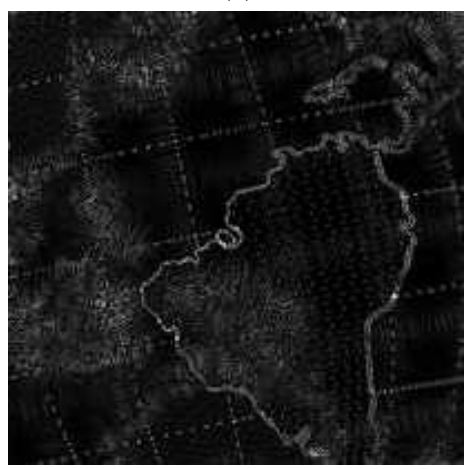

(e)

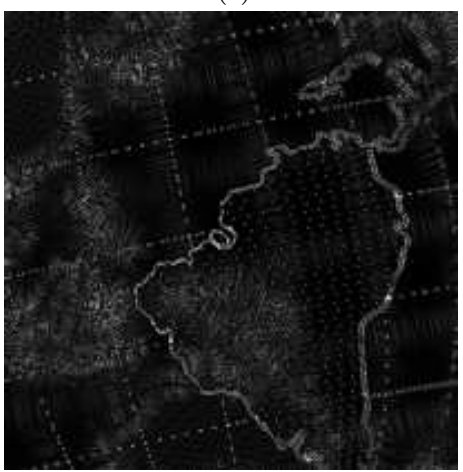

(b)

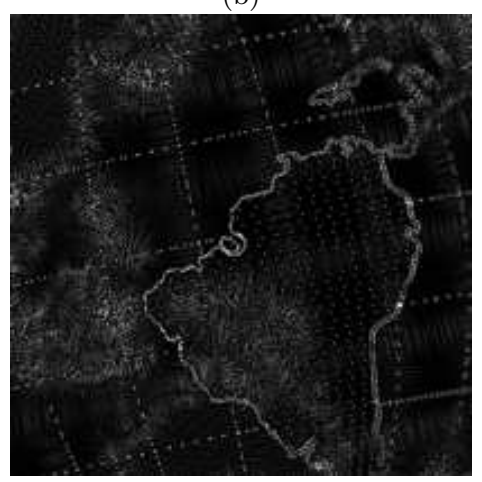

(d)

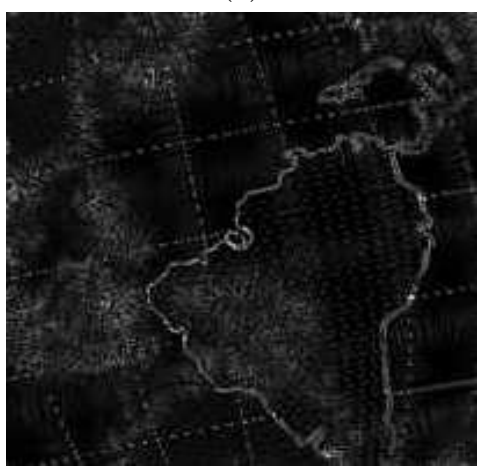

(f)

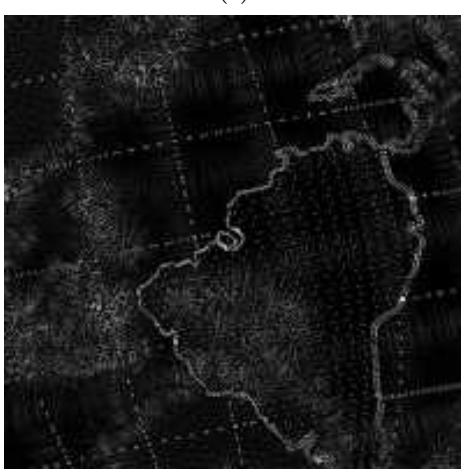

Fig. 5: Visual comparison of cropped region in Earth8KS decoded at ((c)-(d)) 0.1 bps and ((e)-(f)) 0.4 bps with our method (left) and HEVC (right). Even if HEVC's PSNRs values are superior to those provided by our method, the latter leads to better visual quality due to the fact that fine quantizer selection has been used for the cropped region. 
(a)

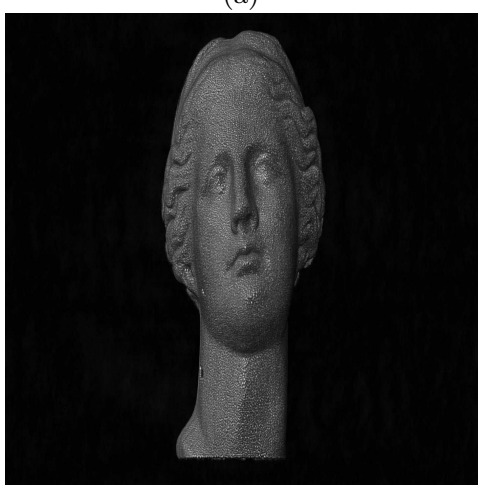

(c)

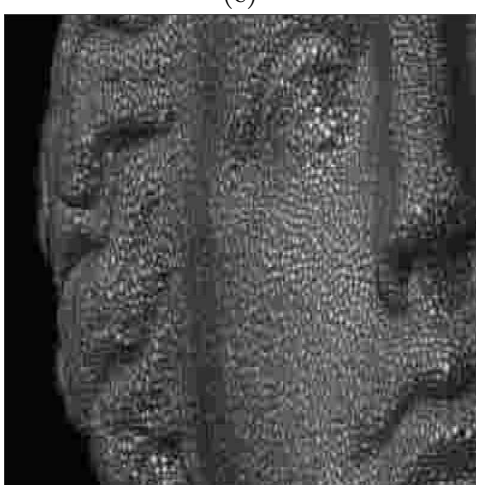

(e)

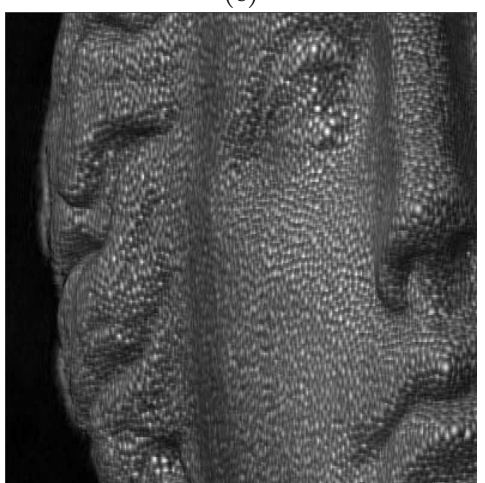

(b)

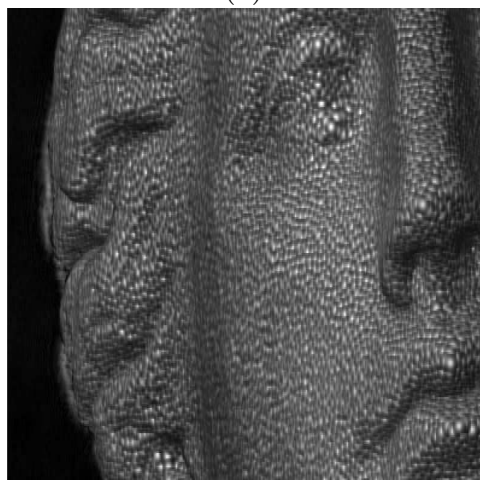

(d)

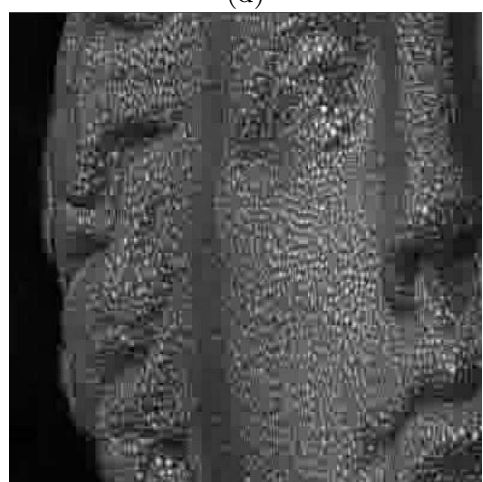

(f)

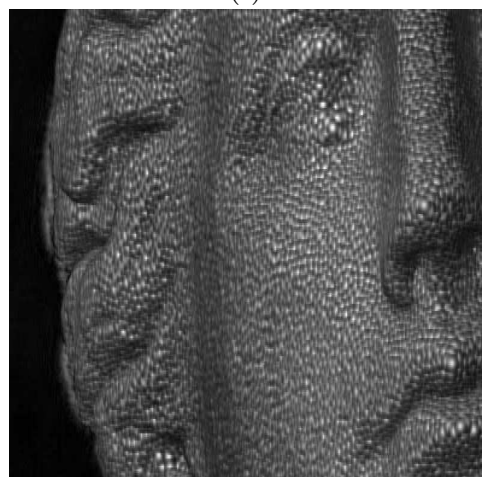

Fig. 6: Visual comparison of cropped region in Venus decoded at ((c)-(d)) 0.2 bps and ((e)(f)) $0.8 \mathrm{bps}$ with our method (left) and HEVC (right). Even if HEVC's PSNRs values are superior to those provided by our method, the latter leads to better visual quality due to the fact that fine quantizer selection has been used for the cropped region. 
- PSNR measure is not a sufficient criterion to evaluate the reconstructed hologram quality. We can see that HEVC provides the highest PSNR, while the perceived quality is much poorer than that of the proposed method (relatively large ringing artefacts).

- Our method is backward compatible with JPEG standard, while yielding very competitive. compression performance and reconstruction quality with respect to HEVC and SPIHT codecs.

- For the PSDHs extracted from Interfere-II database, our method delivers better compression performance results than those obtained for PSDHs extracted from Interfere-I database. This is means that our method is well adapted to PS holograms of high resolution.

- By considering the NRMS results, we found to our great surprise that our method ouperforms HEVC, for 2D Dice, 3D Venus, 3D Multi, Venus8KS and Ball8KS and produces very similar results for the other holograms.

Discussing the above mentioned findings, it is immediately clear that the use of region based bit allocation procedure in conjunction with JPEG algorithm yields satisfactory compression improvements. Specially, significant gain in compression performance over HEVC is attainable when NRMS metric is considered. The increased performance can be explained by the use of finer quantizers on the ROI. This leads to the preservation of the most important details on the object region, which in turn allows convenient recovery in the hologram domain, after performing the inverse reconstruction on the decoded bitsream. Furtheremore, our compression system inherently supports backward and forward compatibility with legacy JPEG formats, which is not the case for wavelet-based codecs. Given this fact and knowing that JPEG format is today the most popular standard and that its ecosystem is strong and will most likely continue to grow in the foreseeable future, we believe that wavelet-based coding approaches for digital holograms are perhaps not the best design option, and that our encoder is a good candidate in applications like immersive browsing and virtual reality applications.

As a final note, we mention that it is utmost important to take into consideration the characteristics and limitations of the different encoding paradigms, and that one should carefully decide about the proper design methodology depending on the required functionality of the codec that is to be implemented.

\section{Conclusions}

In this paper we bring a deep analysis of highly efficient image compression techniques using digital holographic data. Doing so, we explore the efficiency of a DCT-based coding scheme, representing an alternative to the well known JPEG coding design. More specifically, we developed an object based coding system that uses a Lagrangian-driven rate allocation mechanism on selective regions while still compliant with the JPEG bitstream. Regarding compression efficiency and reconstruction quality, it yields competitive results with waveletbased codecs. 
Finally, we believe that our coding method offers a good balance of backward compatibility to JPEG, file size and reconstruction quality. Therefore, it may serve as an enticing alternative to the current state-of-the-art and that findings acquired from the conducted studies are helpful for realizing new JPEG-based hologram codec in line with the objectives of the upcoming JPEG PLENO standard.

The research done in this work could be continued by investigating the following items:

- Parallel processing: in the hologram domain, phase-shifting holograms are most often represented by two sets of data which are compressed independently in the same way. Thereby, it is interesting to build a compression system, entirely automatic, that uses smart ways to parallelize the compression process for the two data sets.

- Adapt to the context: With the emergence of holographic technology, the embedding of holographic data inside Web applications is a new challenge; the visualization of digital holograms through handheld devices is also emerging. Consequently, compression methods have to adapt to this heterogeneous context by presenting different settings.

- Perceptual metrics: Since the final destination of a compressed hologram is often to be displayed to a human observer, the perceived visual quality should be the criterion to take into account when evaluating a compression algorithm.

\section{Acknowledgments}

The authors are particularly grateful to David Blinder and Peter Schelkens (ETRO-VUB, Brussels, Belgium), Manuella Pereira (University of Beira Interior, Covilha, Portugal), José Peixeiro (Técnico Lisboa, Portugal) and Patrick Gioia (Orange Labs, Rennes, France) for their support, the very interesting discussions and for giving us access to their holographic display.

\section{References}

1. Okoshi T.: 'Three-Dimensional Imaging Techniques', New York: Academic Press, 1976.

2. Leith E. N., Upatnieks J.: 'Reconstructed wavefronts and communication theory', Journal of the Optical Society of America, 1962. 52(10), pp. 1123-1128.

3. Yamaguchi I.,Zhang T.:'Phase shifting digital holography', Optics letters, 1997.

4. Gabor D.: 'A new microscopic principle', Journal of Nature, 1948, 161.

5. Yaras F., Kang H., Onural L.: 'State of the art in holographic displays: A survey', IEEE Journal of Display Technology, 2010, 6, pp. 443-454.

6. Schnars U., and Juptner W.,: 'Digital recording and numerical reconstruction of holograms', Measurement Science and Technology, 2002, pp. 85-101.

7. Kurbatova E.A., Cheremkhin P.A., Evtikhiev N.N., Krasnov V.V., Starikov S.N.: 'Methods of Compression of Digital Holograms', Physics Procedia, 2010, 73, pp. 328-332.

8. Tricoles G. p.: 'Computer generated holograms: an historical review', Applied Optics, 1987, 26, pp. 4351-4360. 
9. Symeonidou A., Blinder D., Munteanu A., Schelkens P., 'Computer-generated holograms by multiple wavefront recording plane method with occlusion culling', Optics Express, 2015, 23, , pp. 22149-22161.

10. Darakis E., Naughton T., Soragha J., Javidi B.: 'Measurement of compression defects in phase-shifting digital holographic data', Proceedings of SPIE, Optical Information Systems IV, 6311, San Diego, California, US, 2013.

11. Xing Y., Pesquet-Popescu B., Dufaux F.: 'Comparative study of scalar and vector quantization on different phase-shifting digital holographic data representations', 3DTVConference: The True Vision - Capture, Transmission and Display of $3 D$ Video, Budapest, Hungry, 2014.

12. Dufaux F., Xing Y., Pesquet-Popescu B., Schelkens P.: 'Compression of digital holographic data: an overview', SPIE Proc. Applications of Digital Image Processing XXXVIII, San Diego, CA, USA, Aug. 2015.

13. Xing Y., Kaaniche M., Pesquet-Popescu B., Dufaux F.: 'Digital holographic data representation and compression', Academic Press, Oct. 2015.

14. Blinder D., Ahar A., Symeonidou A., Xing Y., Bruylants T., Schreites C., PesquetPopescu B., Dufaux F., Munteanu A., Schelkens P.: 'Open access database for experimental validations of holographic compression engines', Proc. 7th International Workshop on Quality of Multimedia Experience (QoMEX'2015), Messinia, Greece, May 2015.

15. Peixeiro J., Brites C., Ascenso J., Pereira F.: 'Digital holography: Benchmarking coding standards and representation formats', IEEE International Conference on Multimedia and Expo (ICME), Seattle, WA, USA, 2016.

16. Bernardo M., Fernandes P., Arrifano A., Antonini M., Fonseca E., Fiadeiro P., Pinheiro A., Pereira M.: 'Holographic representation: Hologram plane vs. object plane', Signal Processing: Image Communication, 2018, 68, pp. 193-206.

17. JPEG PLENO Abstract and Executive Summary, ISO/IEC JTC 1/SC 29/WG1 N6922, Sydney, Australia, 2015.

18. Ratnakar V., Livny M.: 'RD-OPT : An efficient algorithm for optimizing DCT quantization tables', IEEE Processings of the Data Compression Conference (DCC), Snowbird, UT, USA, Mar. 1995, pp. 332-341.

19. Schnars U., Juptner W.: 'Digital recording and numerical reconstruction of holograms', Measurement Science and Technology, 2002, 13, pp. 85-101.

20. Goodman J.W.: 'Introduction to Fourier Optics' (Roberts and Company Publishers, 1979 , 3rd edn.)

21. Xing Y., Kaaniche M., Pesquet-Popescu B., Dufaux F.: 'Vector lifting scheme for phaseshifting holographic data compression', Optical Engineering, 2014, 53, pp. 98-109.

22. Naughton T. J., Frauel Y., Javidi B., Tajahuerce E.: 'Compression of digital holograms for three-dimensional object reconstruction and recognition', Optics Express, 2002, 41, pp. 4124-4132.

23. Mills G.A., Yamaguchi I.: 'Effects of quantization in phase-shifting digital holography', Applied Optics, 2005, 44, pp. 1216-1225.

24. Naughton T.J., McDonald J.B., Javidi B: 'Efficient compression of fresnel fields for internet transmission of three-dimensional images', Applied Optics, 2003, 42, pp. 47584764.

25. Shortt A.E., Naughton T.J., Javidi B.: 'A companding approach for nonuniform quantization of digital holograms of three-dimensional objects', Optics Express, 2006, 14, pp. 5129-5134.

26. Arrifano A., Antonini M., Pereira M.: 'Multiple description coding of digital holograms using Maximum-a-Posteriori', IEEE 4th European Workshop on Visual Information Processing (EUVIP), Paris, France, 2013.

27. Liebling M., Blu T., Unser M.: 'Fresnelets: new multiresolution wavelet bases for digital holography', IEEE Trans. Im. Proc., 2003, 12, pp. 29-43.

28. Darakis E., Soraghan J.: 'Use of fresnelets for phase-shifting digital hologram compression', IEEE Trans. Im. Proc., 2006, 15, pp. 3804-3811, Apr. 2006.

29. Said A., Pearlman W.: 'A new fast and efficient image codec based on set partitioning in hierarchical trees', IEEE Trans. Circuits Syst. Video Technol., 1996, 6, pp. 243-250.

30. Viswanathan K., Gioia P., Morin L., 'Morlet wavelet transformed holograms for numerical adaptive view-based reconstruction', Proceedings of SPIE, Optics and Photonics for Information Processing VIII, 9216, San Diego, California, US, 2014. 
31. Viswanathan K., Gioia P., Morin L., 'Wavelet compression of digital holograms: towards a view-dependent framework', Proceedings of SPIE, Applications of Digital Image Processing XXXVI, 8856, San Diego, California, US, 2013.

32. Daubechies I. Jaffard S., Journes J.L.: 'A simple wilson orthonormal basis with exponential decay', SIAM Journal of Mathematics, 1991, 22, pp. 554-573.

33. Skodras A., Christopoulos C., Ebrahimi T.: 'The JPEG 2000 still image compression standard', IEEE Signal Processing Magazine, 2001, 18, pp. 38-58.

34. Viswanathan K., Gioia P., Morin L.: 'A framwork for view-dependent hologram representation and adaptive reconstruction', IEEE International Conference on Image Processing (ICIP), Quebec, Canada, 2015.

35. Wallace G. K., 'The JPEG still picture compression standard, Communications of the ACM, 1991, 34, pp. 30-44.

36. Darakis E., Soraghan J.: 'Compression of interference patterns with application to phase-shifting digital holography', Applied Optics, 2006, 45, pp. 2437-2443.

37. Darakis E., Soraghan J.: 'Compression of phase-shifting digital holography interference patterns', Applied Optics, in Photon Management II, J. T. Sheridan and F. Wyrowski, eds., Proc. SPIE, 2006, 6187.

38. Darakis E., Soraghan J.: 'Reconstruction domain compression of phase-shifting digital holograms', Applied Optics, 2007, 46(3), pp. 351356.

39. Taubman D.: 'High performance scalable image compression with EBCOT', IEEE Trans. Im. Proc., 2000, 9, pp. 1158-1170, Apr. 2006.

40. Xing Y., Kaaniche M., Pesquet-Popescu B., Dufaux F.: 'Adaptive non separable vector lifting scheme for digital holographic data compression', Applied Optics, 2015, 54, pp. $98-109$.

41. Goodman J.W.: 'Introduction to Fourier Optics', MaGraw-Hill, 2 edition, 1996.

42. Bruylants T., Blinder D., Ottevaere H., Munteanu A., Schelkens P.: 'Microscopic offaxis holographic image compression with JPEG 2000', Proceedings of SPIE, Optics, Photonics, and Digital Technologies for Multimedia Applications III, 9138, San Diego, California, US, 2014.

43. Graham R.L.: 'An Efficient Algorithm for Determining the Convex Hull of a Finite Planar Set', Information Processing Letters, 1972, 1, pp. 132-133;

44. Sullivan G.J., Bjontegaard G., Luthra A.: 'Overview of the H.264 AVC Video Coding', IEEE Trans. Cir. and Sys.for Video Tech., 2003, 13, pp. 560-576.

45. El Rhammad A., Gioia P., Antonini G., Cagnazzo M., Pesquet B.: 'Color digital hologram compression based on matching pursuit', Applied Optics, 2018, 57(19), pp. 1-13.

46. Sullivan G. J., Ohm J.-R., Han W.-J., Wiegand T.: 'Overview of the High Efficiency Video Coding (HEVC) Standard', IEEE Trans. Cir. and Sys.for Video Tech., 2012, 22 pp. $1649-1668$.

47. Munteanu A., Cornelis J., Der Auwera G. V., Cristea P.: 'Wavelet image compression - the quadtree coding approachs', IEEE Trans. Inf. Technol. Biomed., 1999, 3, pp. $176-185$.

48. Canny J.: 'A Computational Approach To Edge Detection', IEEE Trans. Pattern Analysis and Machine Intelligence., 1986, 8(6), pp. 679-698.

49. Chamakhi N, Bouzidi I., Ouled Zaid A., Dufeaux F.: 'JPEG based Compression of Digital Holograms', IEEE European Workshop on Visual Information Processing, Tampere, Finland, 2018. 